\title{
Mesenchymal Stem Cells: A Novel Therapeutic Approach to Enhance Protective Immunomodulation and Erythropoietic Recovery in Malaria
}

\author{
Meenu Kalkal ${ }^{1} \cdot$ Mrinalini Tiwari $^{2} \cdot$ Reva S. Thakur $^{1} \cdot$ Vikky Awasthi $^{1} \cdot$ Veena Pande $^{3} \cdot$ Debprasad Chattopadhyay $^{4,5}$. \\ Jyoti Das ${ }^{1}$ (1)
}

Accepted: 24 May 2021 / Published online: 12 June 2021

(c) The Author(s), under exclusive licence to Springer Science+Business Media, LLC, part of Springer Nature 2021

\begin{abstract}
Mesenchymal stem cells (MSCs) are self-renewing, multi-potent heterogeneous stem cells that display strong tissue protective and restorative properties by differentiating into cells of the mesodermal lineages. In addition to multi-lineage differentiation capacity, MSCs play important roles in regulating immune responses, inflammation, and tissue regeneration. MSCs play a role in the outcome of the pathogenesis of several infectious diseases. A unique subset of MSCs accumulates in secondary lymphoid organs during malaria disease progression. These MSCs counteract the capacity of malaria parasites to subvert activating co-stimulatory molecules and to regulate expression of negative co-stimulatory molecules on T lymphocytes. Consequently, MSCs have the capacity to restore the functions of CD $34^{+}$haematopoietic cells and $\mathrm{CD} 4^{+}$and $\mathrm{CD} 8^{+} \mathrm{T}$ cells during malaria infection. These observations suggest that cell-based therapeutics for intervention in malaria may be useful in achieving sterile clearance and preventing disease reactivation. In addition, MSCs provide host protection against malaria by reprogramming erythropoiesis through accelerated formation of colony-forming-units-erythroid (CFU-E) cells in the bone marrow. These findings suggest that MSCs are positive regulators of erythropoiesis, making them attractive targets for treatment of malarial anemia. MSC-based therapies, unlike anti-malarial drugs, display therapeutic effects by targeting a large variety of cellular processes rather than a single pathway. In the present review we focus on these recent research findings and discuss clinical applications of MSC-based therapies for malaria.
\end{abstract}

Keywords Mesenchymal stem cells (MSCs) · Haematopoietic stem cells (HSCs) · Malaria · Plasmodium . Immunomodulation $\cdot$ Erythropoiesis $\cdot$ Haematopoiesis

Meenu Kalkal and Mrinalini Tiwari contributed equally to this work.

Jyoti Das

jyoti@mrcindia.org; drjyoti203@gmail.com

1 Parasite-Host Biology, National Institute of Malaria Research, Sector-8, Dwarka, New Delhi 110077, India

2 Department of Genetics and Epigenetics, Institute of Nuclear Medicine \& Allied Science, Timarpur, New Delhi 110054, India

3 Department of Biotechnology, Kumaun University, Nanital 263001, India

4 ICMR Virus Unit, ID \& BG Hospital, Kolkata 700010, India

5 ICMR-National Institute of Traditional Medicine (NITM), Belagavi 590010, India

\section{Introduction}

Malaria caused by Plasmodium parasites is one of the most serious public health problems, which is responsible for an estimated 200-400 million clinical cases annually. According to the recent world malaria report (2020), 0.4 million estimated deaths have been recorded globally in 2019 with an estimated 229 million malaria cases. Approximately twothirds of deaths (67\%) were reported among children aged less than five years [1]. In South-East Asia, India has the highest burden of infection with variation in proportion and severity among different regions. The major clinical manifestations associated with malaria infection include a highly compromised immune system and increased removal of circulating infected and uninfected erythrocytes [2].

The host immune response against malaria parasites is modulated over the course of infection [3,4]. This immune response mainly depends on age, genetic background, and 
previous exposure history of the host along with the biological characteristics of the Plasmodium parasite strain. In general, a potent $\mathrm{T}$ helper 1 (Th1) type pro-inflammatory response to control the parasite load is activated at an early stage of natural infection with Plasmodium parasites. However, the uncontrolled pro-inflammatory response during infection can prolong disease severity and may cause tissue pathology and organ failure $[5,6]$. The detrimental effects of inflammatory responses are counter-balanced by various anti-inflammatory cytokines released by regulatory immune cells. A well-regulated balance of pro- and anti-inflammatory cytokines plays a crucial role in determining the outcome of infection, resulting in resistance or susceptibility to malaria disease. Regulatory T cells (Tregs) expand during infection and suppress the Th1 immune response resulting in increased parasitaemia that leads to host pathology. In addition to compromising the immune response, malaria parasites suppress production of erythrocytes and this dyserythropoiesis in the bone marrow results in depletion of the infected as well as uninfected erythrocytes which contributes to malarial anaemia. Additionally, tissue deposition of haemozoin, a byproduct of haemoglobin digestion formed in the haem detoxification process has been linked to malarial anaemia and dyserythropoiesis. Moreover, the proliferation and differentiation of erythroid progenitors are also impaired during malaria infection due to reduced expression of erythroid-specific transcription factors such as GATA-1 and GATA-2 [7-9]. GATA-1, a key transcription factor for erythroid cell development, facilitates the survival and late-stage differentiation of erythroid precursors, whereas GATA-2 is critical for the maintenance and proliferation of haematopoietic progenitors. Reduced expression of GATA-1/GATA-2 transcription factors during Plasmodium infection thus modulates the production of erythroid precursors. Because of the complexities involved in malaria pathogenesis, comprehensive studies involving severe malarial anaemia and immunity associated with disease are needed to gain improved understanding of pathogenic events and related defects. This may offer new strategies to combat the rates of mortality and morbidity.

To minimize mortality and morbidity of malaria, several groups of therapeutic compounds have been tested for their anti-malarial efficacy. The majority of anti-malarial drugs employed target the asexual erythrocytic or blood stage of the parasite. Currently, 7 major drug classes are in use for the treatment of malaria: sesquiterpene lactone endoperoxides, 4-aminoquinolines, 8-aminoquinolines, amino alcohols, antifolates, artemisinin derivates, naphthoquinone, and antibiotics [10]. Among these, artemisinin and its derivatives are the most widely and commonly used antimalarials as they have the capacity to kill a broad range of asexual stage parasites from ring forms to early schizonts. However, the major drawback with artemisinin use is its short half-life ( $1 \mathrm{~h}$ or less). Following administration, there is rapid absorption and elimination of this drug and, therefore, multiple doses of the drug are required for retaining its efficacy $[11,12]$. Currently, artemisinin combination therapy (ACT) is employed in efforts to promote treatment efficacy based on synergism of two or more drugs to eradicate various stages of malaria parasites [13-15]. However, the major hurdle associated with the use of antimalarial drugs is the emergence of multidrugresistant (MDR) parasites [16-18]. Other concerns related to antimalarial drugs include poor bioavailability, low solubility and toxicity [19]. Along with these limitations, another important obstacle in the eradication of malaria is the development of a vaccine to impart long-term protective immunity which requires in-depth understanding of the immune responses directed against the different stage-specific malaria antigens. Furthermore, it will be crucial to evaluate vaccine efficacy across endemic areas. The development of an effective vaccine is mainly hindered by the extensive genetic diversity in the protective surface proteins of the parasite. It has been challenging to identify conserved antigenic regions that can broadly neutralize a wide variety of strains. As a result, only few proteins have been evaluated for potential vaccine development. Thus far, RTS,S/AS01 (RTS,S), which targets the circumsporozoite protein and provides partial protection against malaria in young children [20], is the only approved malaria vaccine.

The poor reliability and availability of the aforementioned antimalarial approaches have prompted researchers worldwide to explore alternative prophylactic and therapeutic approaches. Large numbers of antimalarial compounds are in different stages of preclinical and clinical development. Apart from conventional drugs, cell-based therapies such as adoptive therapy with MSCs are being explored in malaria pathogenesis. Preclinical studies have shown promising results for MSC therapy in a wide variety of parasitic infections, cancer, metabolic disorders, immune disorders and other diseases due to their inherent immunomodulatory capacity by activating both innate and adaptive immunity. Their immunomodulatory functions are mainly put forth through secretion of various cytokines and soluble factors along with different cellular interactions involving $\mathrm{T}$ cells, $\mathrm{B}$ cells, macrophages, dendritic cells (DCs), natural killer (NK) cells, neutrophils, basophils, monocytes and other cell types. Another very important characteristic feature of MSCs is their plasticity, which refers to the potential of these cells to change significantly in response to diverse inflammatory or anti-inflammatory stimuli. Based on these striking properties, MSC-based therapies are being evaluated in multiple clinical trials for autoimmune and inflammatory disorders. The therapeutic potential of MSCs has also been explored in a mouse model of malaria. In malaria, MSCs play a crucial 
role in providing protection against Plasmodium infection by modulating the outcome of immune responses and by reprogramming erythropoiesis.

In this review, we will summarise the potential of MSCs for adoptive immunotherapy of malaria. We will also highlight challenges associated with stem cell-based therapies in the treatment of malaria and future research directions.

\section{Immunomodulatory Aspects of Malaria Pathogenesis}

The pathogenesis of malaria involves a complex interplay of Plasmodium parasite-induced alterations and corresponding immunomodulation in the host. Similar to other infectious diseases, the pathogenesis of malaria pathology is usually early, intense and characterized by pro-inflammatory cytokines. However, the immune response to malaria parasites depends on the stage of infection and varies with Plasmodium species. To understand this complex relationship between the parasite and the host immune response, several studies have focused on immune balance and antiparasitic immunity. After inoculation of sporozoites, the organisms remain for several hours in the skin where they prepare for the hepatic stage of infection. Host immune mechanisms are activated first during the liver stage of Plasmodium infection. However, liver or pre-erythrocytic infection is clinically a silent stage of infection that normally lasts for approximately 10 days in humans and 2 days in rodents. During this stage no visible pathology is observed; however, a protective immune response to eliminate infected hepatocytes is triggered. The T-cell mediated response to the pre-erythrocytic stage and its regulation have not been studied extensively. Cytotoxic $\mathrm{CD}^{+}{ }^{+}$-cells as well as $\mathrm{CD} 4^{+} \mathrm{T}$-cell-dependent antibody responses prevent sporozoite invasion of hepatocytes and mediate effective killing of infected cells. Further, the parasite specific cytotoxic $\mathrm{CD} 8^{+} \mathrm{T}$-cells can recognize parasite antigens such as circumsporozoite protein (CSP), liver-stage antigen 1 (LSA-1) and sporozoite threonineasparagine-rich protein ( STARP) presented on MHC class I molecules, which ultimately results in eradication of infected hepatocytes [21]. Additionally, an undefined Th1/Th2 phenotype of $\mathrm{CD}^{+}{ }^{+} \mathrm{T}$-cells has been observed to mediate protection against liver stage infection in murine models of malaria. Th1-derived IFN- $\gamma$ is responsible for host resistance during the pre-erythrocytic stage of infection. IFN- $\gamma$ also upregulates inducible nitric oxide synthase (iNOS) expression in infected hepatocytes, which in turn induces reactive nitrogen intermediates leading to inhibition of intracellular parasite growth. IFN- $\gamma$ also has the capacity to upregulate the cytotoxic activity of $\mathrm{CD} 8^{+} \mathrm{T}$-cells by activating MHC class I expression in infected hepatocytes.
The asexual or erythrocytic stage of disease is responsible for disease pathology. This stage requires a strictly regulated cell-mediated and adaptive immune response to restrict replication of the Plasmodium parasites. At this stage of the disease process an array of signals are responsible for activation of both the innate and adaptive host immune systems. Cells of the innate immune component are activated first in response to whole parasites and/or parasite products (DNA, hemozoin, and the glycosylphosphatidylinositol anchors of malaria proteins) released by ruptured infected erythrocytes. Furthermore, rupture of the Plasmodium-infected erythrocytes and the release of putative malaria toxins also promote the activation of parasite-specific $\mathrm{CD} 4^{+} \mathrm{T}$-cells [22]. These activated $\mathrm{CD} 4^{+} \mathrm{T}$-cells orchestrate a distinct cytokine production pattern with diverse functional capacity following infection of parasitized erythrocytes. As red blood cells do not express MHC class I or II molecules, it is difficult to anticipate the target of Th1 or Th2 T-cells. However, the differentiation pattern of Th1 or Th2 cells is predominantly dependent on the microenvironment during $\mathrm{CD} 4^{+}$ T-cell activation as well as signals generated by antigenpresenting cells (APCs). During the erythrocytic stage of infection, DCs are involved in effective priming of T-cells. When activated in the context of polarizing cytokines, the $\mathrm{CD}^{+}{ }^{+} \mathrm{T}$-cells differentiate into functionally distinct subsets. IFN- $\gamma$, the signature cytokine of Th1 cells expressing the transcription factor T-bet, is critical for controlling the acute erythrocytic stage of infection. In addition, IL-12 is also critical for the induction of protective immune responses. The available experimental data suggest a role of IFN- $\gamma$ in activating macrophages, which is considered an important mechanism of action of this cytokine leading to opsonization of infected red blood cells (RBCs). IFN- $\gamma$ also induces macrophage colony-stimulating factor (M-CSF) and plays a key role in the function and antigen-presenting capacity of myeloid cells. Along with T-bet ${ }^{+}$Th1 cells, IFN- $\gamma$ is also secreted by NK1 cells, NKT cells and $\gamma \delta$ T-cells [23]. Elevated IFN- $\gamma$ also results in overexpression of toll-like receptors (TLRs) by host cells. These TLRs, along with their adaptor signaling molecule MyD88, can recognize a variety of plasmodium-derived components [24]. TLRs are also essential for the generation of the initial pro-inflammatory response during blood-stage of infection, ultimately resulting in systemic pathology and malarial symptoms such as the onset of fever. Another Th1-cell associated cytokine, IL-2, is critical in activating NK-cells which mediate direct cytotoxicity against infected erythrocytes. On the other hand, several studies have also reported pathological roles of Th1 cells and IFN- $\gamma$ during Plasmodium infection. For example, uncontrolled expression of IFN- $\gamma$ results in atypical memory B-cell formation, leading to impairment of humoral immunity. 
The early activation of Th1 cells thus controls parasitemia through secretion of cytokines and effector molecules by cells such as macrophages and NK-cells, which is followed by Th2 responses that induce humoral immune responses to aid in clearing the pathogen. Th2 cells are primarily characterized by secretion of cytokines such as IL- 4 and IL- 5 and expression of the transcription factor GATA-3. IL-4 is the defining cytokine of Th2 cells and plays a critical role in promoting B-cell class-switching. Thus, IL-4 is responsible for enhancing humoral immunity during parasite infection. In addition to Th1 and Th2 cytokines, IL-10 has been shown to regulate immune response by suppressing the uncontrolled expression of pro-inflammatory cytokines induced by Th1 cells. Additionally, IL-10 mediates suppression of excessive inflammation by enhancing the expression of immune checkpoint molecules and downregulating the expression of MHC class II and co-stimulatory molecules on APCs.

Recently, another subset of $\mathrm{CD} 4^{+} \mathrm{T}$ cells, T-follicular helper ( $\mathrm{Tfh}$ ) cells, have been reported to produce IL-21, which is critical for the induction of protective humoral immune responses. IL-21, along with inducible T-cell costimulator (ICOS), promotes the activation and maturation of parasite specific B-cells as well as formation of memory B-cells. These factors can also downregulate the expression of BCL-6 and programmed death-1 (PD-1) during formation of the memory B-cell population. While both Tfh and Th1 memory T-cells mediate secondary immune reactions in malaria, Tfh1-like memory cells are less protective as compared to Th1-like memory cells. Additionally, the Th17 subset of $\mathrm{CD} 4^{+} \mathrm{T}$-cells that expresses the transcription factor ROR- $\gamma t$ has gained attention in the field, owing to its role in chronic inflammation. Like Tfh cells, these cells can secrete IL-21, suggesting a role in supporting germinal centre B (GC-B) cell differentiation. They also secrete IL-22, which is essential for protection against inflammatory pathology.

Another major contributor to malaria immunoregulation is $\mathrm{CD}^{+} \mathrm{CD}^{+} 5^{+} \mathrm{FOXP} 3^{+}$regulatory T-cells (Tregs), which are broadly classified into two distinct subsets: thymus-derived Tregs (t-Tregs) and peripherally derived Tregs (p-Tregs). These cells are induced during the erythrocytic stage of malaria infection, following TGF- $\beta$ activation. These cells can regulate adaptive immune responses to prevent immunopathology associated with excessive inflammation and reactivity to self-antigens, thus increasing self-tolerance. They modulate proliferation, cytokine production and survival of effector T-cells. Furthermore, Tregs also regulate the maturation and function of APCs.

Early activation of Th1 responses assists in limiting the growth of malaria parasites through activation of effector cells such as macrophages, DCs, and NK cells. This is followed by a Th2 response that is responsible for complete clearance of parasites by activating humoral immune responses. In addition to T cell-mediated immune responses, antibodies produced by B cells can provide partial protection. Together, these studies demonstrate that the antibodymediated immune response against Plasmodium parasites is crucial for reducing parasite burden but is not sufficient to provide effective control over the disease. Nevertheless, both cellular and humoral immune components are important and necessary for mediating protection against infection.

\section{Mesenchymal Stem Cells (MSCs) as an Immunotherapy for Malaria}

\section{MSCs: Properties, Immunophenotype and Therapeutic Potential}

MSCs are a heterogenous population of cells, characterized by their capacity to differentiate into a variety of mesodermal lineages such as adipocytes, chondrocytes, and osteoblasts, thus promoting tissue protection and restoration. Their clonogenic potential and multipotency to differentiate into different cell lineages is decided by the in vivo environment. Apart from their ubiquitous presence in the bone marrow, these cells are found in the connective tissue of almost every organ such as skeletal muscle, adipose tissue, umbilical cord, circulatory system, dental pulp, and amniotic fluid, as well as fetal blood, liver, bone marrow, and lungs [25]. In each tissue, a distinct set of surface antigens is expressed by MSCs, which imparts phenotypic heterogeneity and also enhances tissue repair and regeneration through the secretion of various soluble factors. The capacity of MSCs to alter the tissue microenvironment and to display multi-potential activities makes them suitable candidates for the development of effective stem cell-based regenerative medicines. Figure 1 represents some of the important mechanisms underlying the protective role of MSCs in biological systems.

Phenotypically, MSCs express a large number of cell surface markers such as CD105, CD73, CD44, CD90, CD71, CD73, and Stro-1, together with adhesion molecules such as CD106 (vascular cell adhesion molecule [VCAM]-1), CD166 (activated leukocyte cell adhesion molecule [ALCAM]), intercellular adhesion molecule (ICAM)-1, and CD29 [26-31]. However, expression of these markers may differ depending on the tissue of origin and various immunological conditions. This heterogenous expression of surface markers on MSCs results in substantial diversity in their differentiation potential. Additionally, these cells lack conventional haematopoietic markers such as CD45, CD34, CD11, CD14, endothelial cell marker CD31, and other co stimulatory markers such as CD80, CD86 and CD40 [32].

Over the past few decades, a large number of preclinical and clinical studies have explored MSC-based treatments in many disease conditions such as cancer, Parkinson's 
Fig. 1 Schematic representation of the functions of mesenchymal stem cells (MSCs) in biological systems

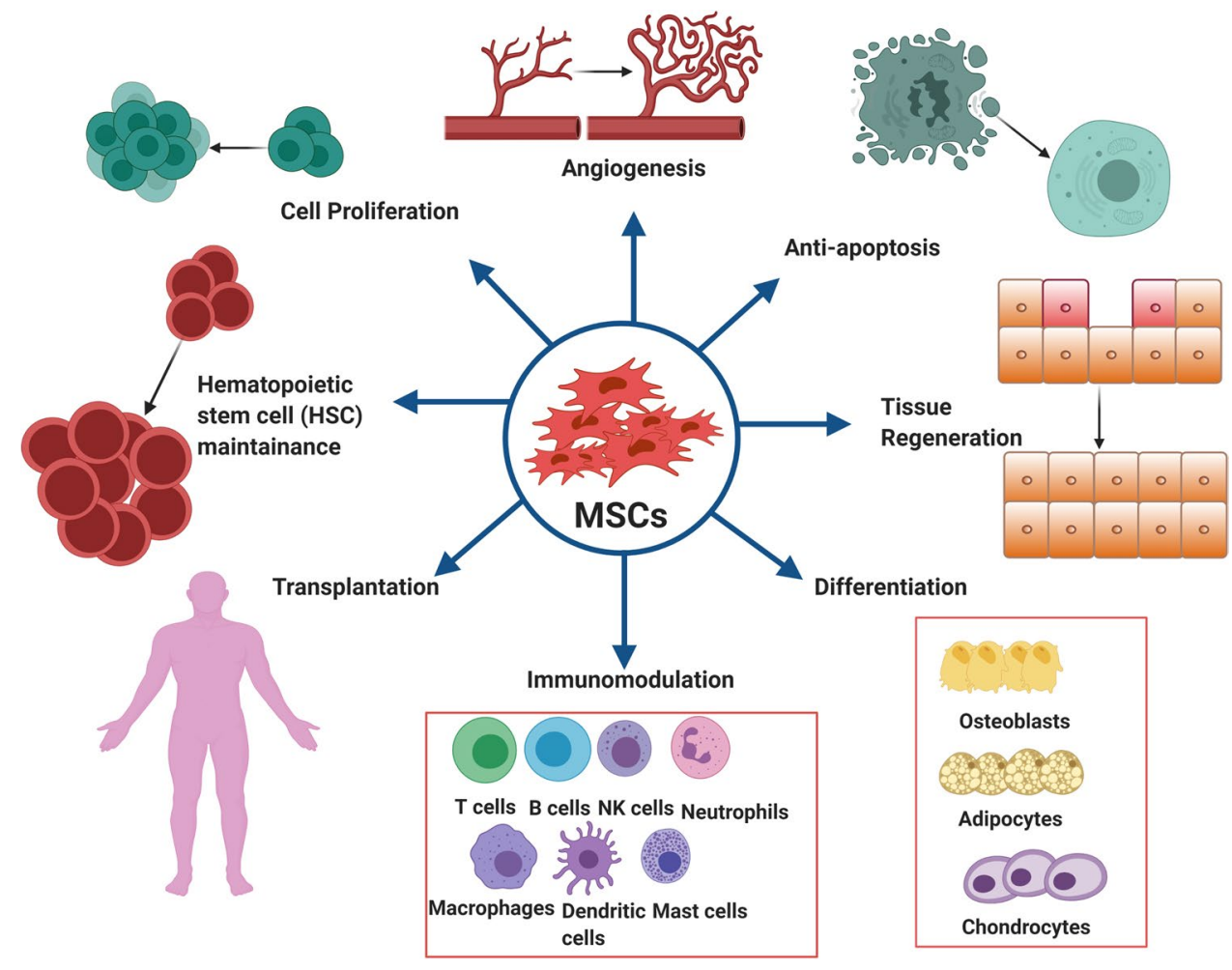

disease, Huntington's disease, transplantation, myocardial infarction, osteogenesis imperfecta, neurological disorders, graft-versus-host disease (GVHD), rheumatoid arthritis, multiple sclerosis, diabetes, and acute respiratory distress syndrome [33-44]. Recently, stem cell therapy has been investigated to treat many parasitic infections and it has become a potential alternative treatment option along with conventional drugs. The therapeutic potential of MSCs is primarily mediated by secretion of various soluble molecules, chemokines, cytokines and growth factors that are produced in response to specific stimuli. Following stimulation, MSCs migrate to the target tissue via the circulation to impart local reparative effects through paracrine secretion of soluble factors and cell-cell contact. Although the exact mechanism of migration is not yet fully understood, it is assumed that various soluble chemokines and their receptors are involved in migration and recruitment of MSCs. One of the most attractive aspects of MSCs is their immunomodulatory potential, which has been extensively studied over the past few years. They are considered potent immunomodulators of the immune system either by cell-cell contact or by secretion of various biologically active molecules, cytokines or growth factors affecting both innate and adaptive immune responses. Recent investigations have provided evidence that MSCs exhibit significant plasticity in their immunomodulatory properties, which has been attributed to the level and nature of inflammation in their microenvironment [45, 46]. Thus, in different disease conditions, the immunoregulatory roles of MSCs are determined by the inflammatory niche that ultimately affects their therapeutic potential. A better understanding of the plasticity of MSC-mediated immunoregulation may help to develop therapeutic regimens.

The ubiquitous presence of MSCs throughout the body, together with the ease of their isolation and their rapid capacity to expand, have expedited the use of these cells as a treatment of choice in cell-based therapies. MSCs are well known for their capacity to activate the immune response by altering the proliferation, maturation and differentiation capacity of immune cells such as T and B cells. These properties also have encouraged the application of MSC-based therapies of immune-related disorders. MSCs are also considered important mediators of erythropoiesis by altering the bone marrow microenvironment in favour of proliferation and differentiation of precursor cells over committed erythrocytes.

The bone marrow (BM) microenvironment is composed of heterogeneous cell populations of haematopoietic and non-haematopoietic origin that are essential for the survival and maintenance of stem cells. This BM niche includes various cell types such as haematopoietic stem cells (HSCs), MSCs or stromal cells, and other multipotent adult progenitor cells. MCSs produce cytokines and haematopoietic growth factors through an autocrine or paracrine mode that facilitates the proliferation, differentiation and regeneration of HSCs. The major cytokines produced by MSCs include interleukin-6 (IL-6), Fms-related tyrosine kinase 3-ligand 
Flt3-L (FL), stem cell factor (SCF), granulocyte colonystimulating factor (G-CSF), macrophage colony-stimulating factor (M-CSF), granulocyte-macrophage colony-stimulating factor (GM-CSF), thrombopoietin (TPO), C-X-C motif chemokine ligand 12 (CXCL-12), and interleukin-11 (IL-11) $[30,47]$. These cytokines play central roles in haematopoiesis, promoting expansion, proliferation and differentiation of different haematopoietic cell lineages. Haematopoiesis is the cumulative result of various signalling pathways that are mediated by a complex interplay of cytokines and soluble growth factors. Cytokines released by MSCs in conjunction with other factors activate multiple signalling pathways of haematopoiesis, such as Wnt, Notch, Hedgehog and TGF- $\beta$ / SMAD [48, 49].

\section{MSC-Mediated Immunomodulation in Malaria}

Immunomodulation is referred to as a process aimed at modifying the immune response. In malaria, different immune cells are activated at different tissue sites following exposure to both asexual and sexual parasite stages, which ultimately results in hyper activation of the immune response. Host immune responses play a critical role in the initiation, severity and outcome of malaria. Various approaches and advanced treatment options have been employed to modulate the immune system in malaria. In most human studies, administration of conventional single or combination drug therapy has been attempted to modulate malarial immunity. However, cytokines or anti-cytokine antibodies, antibodies against adhesion molecules, and adjuvant drug therapies also have been explored. Cell-based therapies have emerged as a promising option for a variety of diseases, including malaria. Stem cells in malaria were first evaluated in 1991, showing that multi-potent haematopoietic stem cells increase the survival in Plasmodium berghei infected mice [50]. This work prompted studies with MSCs isolated from secondary lymphoid organs of $P$. berghei infected mice, which conferred host resistance against malaria upon adoptive transfer through enhanced production of the pro-inflammatory cytokines IL-6, IL-12 and TNF- $\alpha$, and suppression of IL-10, an anti-inflammatory cytokine. Furthermore, in this model, MSCs also inhibit the accumulation of malaria parasite-induced Tregs which are the key mediators of immune responses and whose numbers increase during human or murine malaria [6, 51]. In addition, MSCs hinder the induction of the inhibitory co-stimulatory receptor programmed death-1 (PD-1) by T cells upon infection with malaria parasites [52]. Moreover, Plasmodium infection results in depletion of $\mathrm{CD}^{+}$and $\mathrm{CD} 8^{+} \mathrm{T}$-cells, and infusion of MSCs from malaria-infected animals restores the proliferation of $\mathrm{CD}^{+}$and $\mathrm{CD} 8^{+}$T-cells (Fig. 2). MSCs induced during malaria infection are known to exhibit inflammatory properties, as these cells were unable to produce NO. As a consequence, it was suggested that MSCs induced during malaria infection exhibit inflammatory properties and promote cellular immune responses that protect against malaria. In another study of experimental cerebral malaria (ECM) the protective effects of BM-MSC therapy in multiple organ dysfunction were evaluated. Infusion or transplantation of BM-MSCs resulted in reduction of parasitaemia and malaria
Fig. 2 Mesenchymal stem cells mediate protective immunomodulation during murine malaria

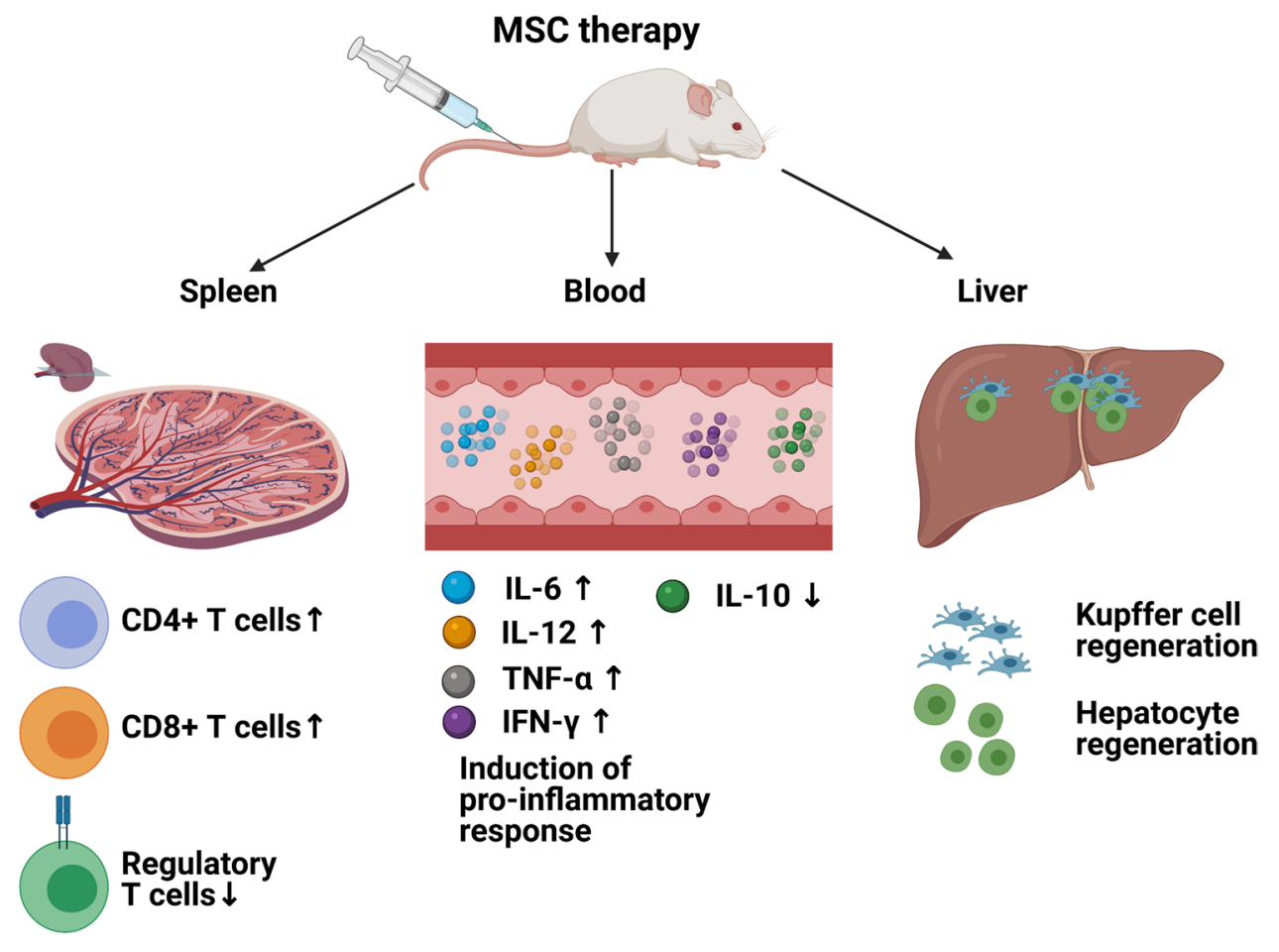


pigment deposition along with an increase in $\mathrm{CD} 11 \mathrm{~b}^{+}$cells in spleen. Additionally, an increase in the number of tissueprotective astrocytes and oligodendrocytes in BM-MSCtreated mice was observed, implicating their role in tissue repair [53]. Although the inflammatory process remained unaffected, there was a substantial increase in phagocytic neutrophils in the brain [54]. Further, there was an increase in hepatocytes and Kupffer cell regeneration in mice that might be due to the inherent regenerative ability of MSCs. Surprisingly, these alterations did not appear to be associated with changes in the expression of pro-inflammatory cytokines, including IFN- $\gamma$, TNF- $\alpha$, VEGF, and chemokine (C-X-C motif) ligand 1 (CXCL1) post-infection in lungs of BM-MSC-treated mice. Although this study explained the protective role of BM-MSCs by increasing host survival, the duration of observation in this study was relatively short. Therefore, evaluation of the effect of MSC therapy over an extended time period during Plasmodium parasite infection may provide more insight before considering clinical trials.

The potential capacity of MSCs to synergize with currently available antimalarials should also be explored. A recent study showed that combined treatment of MSCs with chloroquine, using a mouse model of cerebral malaria, increased the adherence of leukocytes in the brain microvasculature and resolved depression and anxiety like-behaviours [55].

\section{MSC-Mediated Regulation of Erythropoiesis in Malaria}

Various mediators produced by host adaptive immune responses as well as metabolites secreted by the parasite are responsible for pathological conditions associated with malarial anemia. Ineffective erythropoiesis and dyserythropoiesis are major contributing factors responsible for worsening the condition during Plasmodium infection. Dyserythropoiesis results in the increased removal of circulating infected and uninfected erythrocytes and decreased production of mature erythrocytes from the bone marrow due to defective development of erythrocytes that eventually suppresses the normal erythropoietic responses [56, 57]. As a consequence of infection and hemolysis of infected erythrocytes, severe malarial anemia develops that compromises recovery from infection, ultimately resulting in increased morbidity and mortality [58]. During the process of hemolysis, host hemoglobin is degraded by Plasmodium proteases and converted into hematin, which is toxic to the parasite. To overcome this toxicity of hematin, the Plasmodium parasites developed a mechanism of detoxification by converting hematin to non-toxic insoluble hemozoin [59]. The total amount of hemozoin accumulated in the host is indicative of parasite load that is potentially responsible for dyserythropoiesis and decreased erythrocyte production. A recent report has provided support for MSC-mediated regulation of erythropoiesis in a mouse model of malaria [6]. Hemozoin levels seem to be directly associated with parasite load and therefore reduced levels of hemozoin after infusion of MSCs are a clear indication of reduced parasitaemia. Reduction of hemozoin content strongly suggests the potential of MSCs to improve erythropoiesis.

In addition to dyserythropoiesis, the erythropoietic response is characterised by altered erythroid precursors. Erythropoiesis is a well-regulated process in which erythroid progenitors proliferate and differentiate to ultimately generate RBCs in the circulation. It is a multi-step process whereby bone marrow haematopoietic stem/progenitor cells (HSPC) differentiate into committed erythroid progenitors generating colony-forming unit-granulocyte, -erythrocyte, -monocyte, and -megakaryocyte (CFU-GEMM) cells. CFU-GEMM then differentiate into more committed burstforming unit-erythroid (BFU-E) cells. BFU-E are the earliest progenitors exclusively committed to the erythroid lineage to become colony-forming unit-erythroid (CFU-E) cells. Mature erythrocytes are generated when CFU-E enter into a terminal differentiation process: pro-erythroblasts $\rightarrow$ eryth roblasts $\rightarrow$ reticulocytes $\rightarrow$ erythrocytes. A number of in vivo studies explain the erythropoietic response following infection by murine Plasmodium species. Generally it has been observed that erythroblasts, BFU-E, and CFU-E numbers decrease within $24 \mathrm{~h}$ after infection with $P$. berghei [60]. A similar study with Plasmodium yoelii 17XL also showed a decline in the BFU-E population in bone marrow [61]. A recent study showed increased numbers of CFU-E colonies and decreased numbers of BFU-E colonies in mice infused with MSCs using in vitro colony forming assays [52]. This observation suggests increased differentiation of BFU-E towards more mature CFU-E, which is indicative of enhanced erythropoiesis in the presence of MSCs (Fig. 3). Thus, MSCs act as positive regulators of erythropoiesis, making them attractive targets for treatment of malarial anemia. Moreover, malaria infection leads to reduced expression of the GATA-1 transcription factor, which likely plays a role in this reduced erythropoiesis. Infusion of MSCs was also found to enhance the expression of GATA-1. GATA1 , commonly referred to as a master transcription factor of the erythroid lineage, plays an important role in the differentiation of BFU-E to CFU-E, which is facilitated by the mediator subunit MED1/TRAP220 [7-9]. By performing in vitro studies, it has been observed that MED1/TRAP220 binds to GATA factors that forms a bridge between these transcription factors and the RNA polymerase II machinery [7]. Further studies are needed to determine the mechanisms involved.

MSCs regulate the generation of HSCs and their capacity to differentiate into committed cell lineages through direct cell-cell contact and secretion of various cytokines [62]. To study the dynamic balance of the haematopoietic 
Fig. 3 Mesenchymal stem cells involved in reprogramming of malaria-induced dyserythropoiesis by enhancing the differentiation of erythroid progenitors

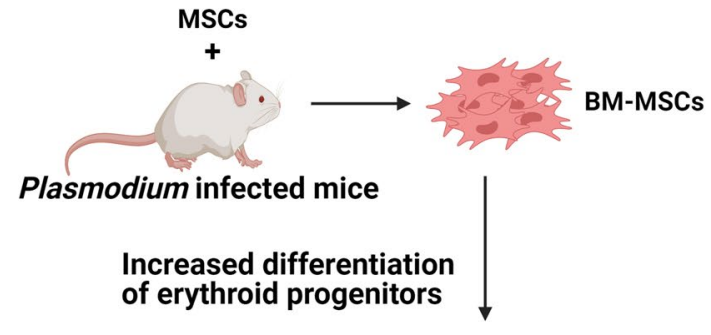

CFU-E $\uparrow$ BFU-E $\downarrow$

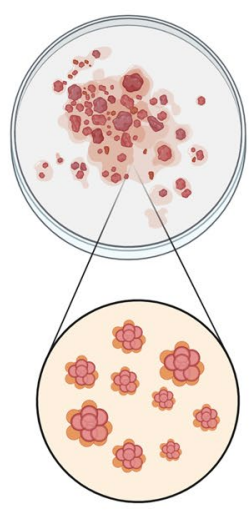

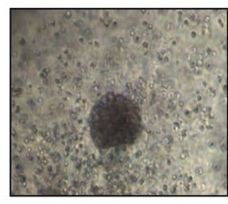

BFU-E

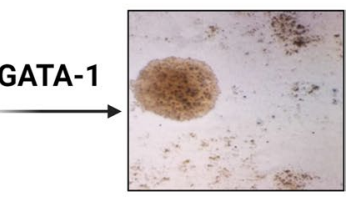

CFU-E compartment, Belyaev et al. (2010) reported the existence of a Lin ${ }^{-} \mathrm{IL}-7 \mathrm{R} \alpha^{+} \mathrm{c}-\mathrm{Kit}^{\mathrm{hi}}$ progenitor cell subset that has both myeloid and lymphoid potential under in vitro culture conditions. Transplantation of these cells induced atypical IL-7R $\alpha^{+} \mathrm{c}-\mathrm{Kit}^{\mathrm{hi}}$ progenitor cells in Plasmodium chabaudi infected mice, which upregulated the proliferation of myeloid biased progenitors that contributed to clearance of parasite-infected erythrocytes. The expansion of these progenitor cells appeared to be critically dependent on IFN- $\gamma$ signalling [63]. Another study showed that MSCs make a major contribution to haematopoietic progenitor cell proliferation and differentiation in malaria by enhancing the differentiation of $\mathrm{CD} 34^{+}$cells. Since $\mathrm{CD} 34^{+}$HSCs have an intrinsic capacity to give rise to all blood cells, this mechanism might contribute to the repair of malaria parasite-induced tissue injury [52]. Moreover, cytokines such as IL-12 that are responsible for self-renewal and differentiation of multipotent progenitor cells are also induced.

While preclinical studies have raised enthusiasm for MSCbased therapies of malaria, translation of these findings to human patients will require studies to determine the best source for MSC isolation and to explore the ultimate fate of these cells after infusion. Figure 3 provides a proposed experimental design to study the possible role of MSCs in reprogramming erythropoiesis.

\section{Conclusions and Future Prospects}

Stem cell therapy is an emerging treatment for a wide variety of human disorders. It has also been successfully introduced in the treatment of many fatal parasitic infections posing a global health threat. In malaria, attempts at stem cell therapy were initiated in the early 90's and the advantages of employing MSCs were subsequently realized. The multifaceted and versatile properties of MSCs in mediating protective immune responses and maintaining the haematopoietic niche have attracted the exploration of MSCs as a therapeutic approach for malaria-induced dyserythropoiesis and immune alterations. The role of MSCs as niche cell organizers and immunomodulators provides both opportunities and challenges in the development of stem cell-based therapies.

A comprehensive analysis of the mechanisms by which MSCs can protect against malaria should be performed. Thorough evaluation is required to explore the clinical effectiveness of MSCs in malaria infection by various strains of Plasmodium parasites. It will also be important to pursue the pattern and impact of protection using primed as well as naive MSCs. This approach will enable us to obtain a better understanding of the capacity of MSCs to modulate immune responses and eventually optimize protocols for their ex vivo expansion for clinical use.

As a variety of exogenous factors may greatly impact the biological properties and efficacy of MSCs, it is critical to obtain a better understanding of the fate of MSCs following their infusion into the host. Along with this, for improved clinical efficacy, it is also important to have indepth knowledge of the dose as well as route of administration. Appropriate evaluation of efficacy at both preclinical and clinical levels will help to determine the therapeutic potential of MSCs when administered to patients. With a deeper understanding of the immunomodulatory properties and impact on erythropoiesis by MSCs, it should become 
possible to establish a new clinical paradigm in malaria research.

Acknowledgements We are thankful to ICMR-National Institute of Malaria Research (ICMR-NIMR) for infrastructural support. Meenu Kalkal is the recipient of a senior research fellowship from Council of Scientific and Industrial Research (CSIR), Govt of India. The authors are highly obliged to Dr. Luc Van Kaer, Professor of Pathology, Microbiology and Immunology at Vanderbilt University Medical Center, Tennessee, USA for his generous support in reading and editing the manuscript.

Author's Contributions MK, MT and JD designed the original idea and developed it in detail, reviewed the literature, and wrote the manuscript. MK generated all the figures and arranged references. RST and VA helped in reviewing the literature. VP and DC provided valuable feedback and helped to revise the manuscript.

Data Availability Not applicable.

Code Availability Not applicable.

\section{Declarations}

Ethics Approval Not applicable.

Consent to Participate Not applicable.

Consent to Publish Not applicable.

Conflict of Interest The authors declare that they have no conflict of interest.

\section{References}

1. World Health Organization. (2020). World malaria report 2020: 20 years of global progress and challenges

2. Haldar, K., \& Mohandas, N. (2009). Malaria, erythrocytic infection, and anemia. Hematology. American Society of Hematology. Education Program, 2009, 87-93.

3. Doolan, D. L., Dobaño, C., \& Baird, J. K. (2009). Acquired immunity to malaria. Clinical Microbiology Reviews, 22(1), 13-36.

4. Langhorne, J., Ndungu, F. M., Sponaas, A. M., \& Marsh, K. (2008). Immunity to malaria: more questions than answers. Nature Immunology, 9(7), 725-732.

5. Scorza, T., Magez, S., Brys, L., \& De Baetselier, P. (1999). Hemozoin is a key factor in the induction of malaria-associated immunosuppression. Parasite Immunology, 21(11), 545-554.

6. Thakur, R. S., Tousif, S., Awasthi, V., Sanyal, A., Atul, P. K., Punia, P., et al. (2013). Mesenchymal stem cells play an important role in host protective immune responses against malaria by modulating regulatory T cells. European Journal of Immunology, 43(8), 2070-2077.

7. Borggrefe, T., Waskow, C., Roeder, R. G., \& Stumpf, M. (2004). Severely impaired erythropoiesis in mice lacking mediator subunit Med1/TRAP220. Blood, 104, 1611-1611.

8. Ohneda, K., \& Yamamoto, M. (2002). Roles of hematopoietic transcription factors GATA-1 and GATA-2 in the development of red blood cell lineage. Acta Haematologica, 108(4), 237-245.
9. Tang, Y., Joyner, C. J., Cabrera-Mora, M., Saney, C. L., Lapp, S. A., Nural, M. V., et al. (2017). Correction to: Integrative analysis associates monocytes with insufficient erythropoiesis during acute Plasmodium cynomolgi malaria in rhesus macaques. Malaria Journal, 16(1), 486.

10. Haldar, K., Bhattacharjee, S., \& Safeukui, I. (2018). Drug resistance in Plasmodium. Nature Reviews Microbiology, 16(3), $156-170$.

11. Kumar, N., \& Zheng, H. (1990). Stage-specific gametocytocidal effect in vitro of the antimalaria drug qinghaosu on Plasmodium falciparum. Parasitology Research, 76(3), 214-218.

12. ter Kuile, F., White, N. J., Holloway, P., Pasvol, G., \& Krishna, S. (1993). Plasmodium falciparum: in vitro studies of the pharmacodynamic properties of drugs used for the treatment of severe malaria. Experimental Parasitology, 76(1), 85-95.

13. WHO Guidelines Approved by the Guidelines Review Committee. (2015). Guidelines for the Treatment of Malaria. World Health Organization Copyright $@$ C World Health Organization 2015.

14. Barber, B. E., Grigg, M. J., William, T., Yeo, T. W., \& Anstey, N. M. (2017). The Treatment of Plasmodium knowlesi Malaria. Trends in Parasitology, 33(3), 242-253.

15 Premji, Z. G. (2009). Coartem: the journey to the clinic. Malar Journal, 8 Suppl 1(Suppl 1), S3.

16. Amato, R., Lim, P., Miotto, O., Amaratunga, C., Dek, D., Pearson, R. D., et al. (2017). Genetic markers associated with dihydroartemisinin-piperaquine failure in Plasmodium falciparum malaria in Cambodia: A genotype-phenotype association study. The Lancet Infectious Diseases, 17(2), 164-173.

17. Ashley, E. A., Dhorda, M., Fairhurst, R. M., Amaratunga, C., Lim, P., Suon, S., et al. (2014). Spread of artemisinin resistance in Plasmodium falciparum malaria. New England Journal of Medicine, 371(5), 411-423.

18. Miotto, O., \& Amato, R. (2015). Genetic architecture of artemisininresistant Plasmodium falciparum. Nature Genetics, 47(3), 226-34.

19. Tse, E. G., Korsik, M., \& Todd, M. H. (2019). The past, present and future of anti-malarial medicines. Malaria Journal, 18(1), 93.

20. RTS, S Clinical Trials Partnership. (2015). Efficacy and safety of RTS, S/AS01 malaria vaccine with or without a booster dose in infants and children in Africa: final results of a phase 3, individually randomised, controlled trial. Lancet, 386(9988), 31-45.

21. Sun, P., Schwenk, R., White, K., Stoute, J. A., Cohen, J., Ballou, W. R., et al. (2003). Protective immunity induced with malaria vaccine, RTS, S, is linked to Plasmodium falciparum circumsporozoite protein-specific CD4+ and CD8+ T cells producing IFN- $\gamma$. The Journal of Immunology., 171(12), 6961-6967.

22. Ing, R., Segura, M., Thawani, N., Tam, M., \& Stevenson, M. M. (2006). Interaction of mouse dendritic cells and malaria-infected erythrocytes: uptake, maturation, and antigen presentation. The Journal of Immunology, 176(1), 441-450.

23. Kurup, S. P., \& Butler, N. S. (2019). T cell-mediated immunity to malaria. Nature Reviews Immunology, 19(7), 457-71.

24. Griffith, J. W., O'Connor, C., Bernard, K., Town, T., Goldstein, D. R., \& Bucala, R. (2007). Toll-like receptor modulation of murine cerebral malaria is dependent on the genetic background of the host. The Journal of infectious diseases., 196(10), 1553-1564.

25. Klimczak, A., \& Kozlowska, U. (2016). Mesenchymal stromal cells and tissue-specific progenitor cells: Their role in tissue homeostasis. Stem Cells International., 2016, 4285215.

26. Conget, P. A., \& Minguell, J. J. (1999). Phenotypical and functional properties of human bone marrow mesenchymal progenitor cells. Journal of Cellular Physiology, 181(1), 67-73.

27. Galmiche, M. C., Koteliansky, V. E., Brière, J., Hervé, P., \& Charbord, P. (1993). Stromal cells from human long-term marrow cultures are mesenchymal cells that differentiate following a vascular smooth muscle differentiation pathway. Blood, 82(1), 66-76. 
28. Haynesworth, S. E., Baber, M. A., \& Caplan, A. I. (1992). Cell surface antigens on human marrow-derived mesenchymal cells are detected by monoclonal antibodies. Bone, 13(1), 69-80.

29. Le Blanc, K., Tammik, C., Rosendahl, K., Zetterberg, E., \& Ringdén, O. (2003). HLA expression and immunologic properties of differentiated and undifferentiated mesenchymal stem cells. Experimental Hematology, 31(10), 890-896.

30. Pittenger, M. F., Mackay, A. M., Beck, S. C., Jaiswal, R. K., Douglas, R., Mosca, J. D., et al. (1999). Multilineage potential of adult human mesenchymal stem cells. Science, 284(5411), 143-147.

31. Sordi, V., Malosio, M. L., Marchesi, F., Mercalli, A., Melzi, R., Giordano, T., et al. (2005). Bone marrow mesenchymal stem cells express a restricted set of functionally active chemokine receptors capable of promoting migration to pancreatic islets. Blood, 106(2), 419-427.

32. Law, S., \& Chaudhuri, S. (2013). Mesenchymal stem cell and regenerative medicine: Regeneration versus immunomodulatory challenges. American Journal of Stem Cells, 2(1), 22-38.

33. Deng, P., Torrest, A., Pollock, K., Dahlenburg, H., Annett, G., Nolta, J. A., et al. (2016). Clinical trial perspective for adult and juvenile Huntington's disease using genetically-engineered mesenchymal stem cells. Neural Regeneration Research, 11(5), 702-5.

34. Götherström, C., \& Walther-Jallow, L. (2020). Stem cell therapy as a treatment for osteogenesis imperfecta. Current Osteoporosis Reports, 18(4), 337-343.

35. Karamini, A., Bakopoulou, A., \& Andreadis, D. (2020). Therapeutic potential of mesenchymal stromal stem cells in rheumatoid arthritis: A systematic review of in vivo studies. Stem Cell Reviews and Reports, 16(2), 276-87.

36. Lee, H. Y., \& Hong, I. S. (2017). Double-edged sword of mesenchymal stem cells: Cancer-promoting versus therapeutic potential. Cancer Science, 108(10), 1939-1946.

37. Lin, F., Ichim, T. E., Pingle, S., Jones, L. D., Kesari, S., \& Ashili, S. (2020). Mesenchymal stem cells as living anti-inflammatory therapy for COVID-19 related acute respiratory distress syndrome. World Journal of Stem Cells, 12(10), 1067-1079.

38. Mendes Filho, D., Ribeiro, P. D. C., Oliveira, L. F., de Paula, D. R. M., Capuano, V., de Assunção, T. S. F., et al. (2018). Therapy with mesenchymal stem cells in Parkinson disease: History and perspectives. The Neurologist, 23(4), 141-147.

39. Miao, C., Lei, M., Hu, W., Han, S., \& Wang, Q. (2017). A brief review: the therapeutic potential of bone marrow mesenchymal stem cells in myocardial infarction. Stem Cell Research \& Therapy, 8(1), 242.

40. Oliveira, A. G., Gonçalves, M., Ferreira, H., \& Neves, M. N. (2020). Growing evidence supporting the use of mesenchymal stem cell therapies in multiple sclerosis: A systematic review. Multiple Sclerosis and Related Disorders, 38, 101860.

41. Si, Y., Zhao, Y., Hao, H., Liu, J., Guo, Y., Mu, Y., et al. (2012). Infusion of mesenchymal stem cells ameliorates hyperglycemia in type 2 diabetic rats: Identification of a novel role in improving insulin sensitivity. Diabetes, 61(6), 1616-1625.

42. van Velthoven, C. T., Kavelaars, A., \& Heijnen, C. J. (2012). Mesenchymal stem cells as a treatment for neonatal ischemic brain damage. Pediatric Research, 71(4 Pt 2), 474-481.

43. Volkman, R., \& Offen, D. (2017). Concise review: Mesenchymal stem cells in neurodegenerative diseases. Stem Cells, 35(8), $1867-1880$.

44. Zhou, X., Jin, N., Wang, F., \& Chen, B. (2020). Mesenchymal stem cells: A promising way in therapies of graft-versus-host disease. Cancer Cell International, 20, 114.

45 Ciavarella, C., \& Pasquinelli, G. (2019). The dual nature of Mesenchymal Stem Cells (MSCs): Yin and Yang of the inflammatory process. Update on mesenchymal and induced pluripotent stem cells. IntechOpen.

46. Wei, X., Yang, X., Han, Z. P., Qu, F. F., Shao, L., \& Shi, Y. F. (2013). Mesenchymal stem cells: a new trend for cell therapy. Acta Pharmacologica Sinica, 34(6), 747-754.
47. Tocci, A., \& Forte, L. (2003). Mesenchymal stem cell: use and perspectives. The Hematology Journal, 4(2), 92-96.

48. Kikuchi, Y., Kume, A., Urabe, M., Mizukami, H., Suzuki, T., Ozaki, K., et al. (2011). Reciprocal upregulation of Notch signaling molecules in hematopoietic progenitor and mesenchymal stromal cells. J Stem Cells Regen Med, 7(2), 61-68.

49. Luis, T. C., Killmann, N. M., \& Staal, F. J. (2012). Signal transduction pathways regulating hematopoietic stem cell biology: introduction to a series of Spotlight Reviews. Leukemia, 26(1), 86-90.

50. Asami, M., Owhashi, M., Abe, T., \& Nawa, Y. (1991). Susceptibility of multipotent haemopoietic stem cell deficient $\mathrm{W} / \mathrm{Wv}$ mice to Plasmodium berghei-infection. Immunology and Cell Biology, 69(Pt 5), 355-360.

51. Hansen, D. S., \& Schofield, L. (2010). Natural regulatory T cells in malaria: host or parasite allies? PLoS Pathogens, 6(4), e1000771.

52. Thakur, R. S., Awasthi, V., Sanyal, A., Chatterjee, S., Rani, S., Chauhan, R., et al. (2020). Mesenchymal stem cells protect against malaria pathogenesis by reprogramming erythropoiesis in the bone marrow. Cell Death Discovery, 6(1), 1-10.

53. Souza, M. C., Silva, J. D., Pádua, T. A., Torres, N. D., Antunes, M. A., Xisto, D. G., et al. (2015). Mesenchymal stromal cell therapy attenuated lung and kidney injury but not brain damage in experimental cerebral malaria. Stem Cell Research \& Therapy, 6(1), 102.

54. Chen, L., Zhang, Z. H., \& Sendo, F. (2000). Neutrophils play a critical role in the pathogenesis of experimental cerebral malaria. Clinical \& Experimental Immunology., 120(1), 125-133.

55. Lima, M. N., Oliveira, H. A., Fagundes, P. M., Estato, V., Silva, A. Y. O., Freitas, R., et al. (2020). Mesenchymal stromal cells protect against vascular damage and depression-like behavior in mice surviving cerebral malaria. Stem Cell Research \& Therapy, $11(1), 367$

56. Chang, K. H., \& Stevenson, M. M. (2004). Malarial anaemia: Mechanisms and implications of insufficient erythropoiesis during blood-stage malaria. International Journal for Parasitology, 34(13-14), 1501-1516.

57. Chasis, J. A., \& Mohandas, N. (2008). Erythroblastic islands: Niches for erythropoiesis. Blood, 112(3), 470-478.

58. Abdalla, S. H. (1990). Hematopoiesis in human malaria. Blood Cells., 16(2-3), 401-16. discussion 17-9.

59. Arese, P., \& Schwarzer, E. (1997). Malarial pigment (haemozoin): A very active "inert" substance. Annals of Tropical Medicine and Parasitology, 91(5), 501-516.

60. Maggio-Price, L., Brookoff, D., \& Weiss, L. (1985). Changes in hematopoietic stem cells in bone marrow of mice with Plasmodium berghei malaria. Blood, 66(5), 1080-1085.

61. Weiss, L., Johnson, J., \& Weidanz, W. (1989). Mechanisms of splenic control of murine malaria: tissue culture studies of the erythropoietic interplay of spleen, bone marrow, and blood in lethal (strain 17XL) Plasmodium yoelii malaria in BALB/c mice. American Journal of Tropical Medicine and Hygiene, 41(2), 135-143.

62. Dazzi, F., Ramasamy, R., Glennie, S., Jones, S. P., \& Roberts, I. (2006). The role of mesenchymal stem cells in haemopoiesis. Blood Reviews, 20(3), 161-171.

63. Belyaev, N. N., Brown, D. E., Diaz, A. I., Rae, A., Jarra, W., Thompson, J., et al. (2010). Induction of an IL7-R(+)c-Kit(hi) myelolymphoid progenitor critically dependent on IFN-gamma signaling during acute malaria. Nature Immunology, 11(6), $477-485$.

Publisher's Note Springer Nature remains neutral with regard to jurisdictional claims in published maps and institutional affiliations. 